\section{Case Reports in Orthopedic Research}

Case Rep Orthop Res 2020;3:94-99

\title{
Hip Arthroscopy in a Baseball Catcher without the Use of a Perineal Post: A Crossover Case Report
}

\author{
Michael P. Hagerty ${ }^{a}$ Nathan W. Skelley \\ aUniversity of Missouri School of Medicine, Columbia, MO, USA; bUniversity of \\ South Dakota Sanford School of Medicine, Department of Orthopedic Surgery, \\ Vermillion, SD, USA
}

\section{Keywords}

Arthroscopy · Femoroacetabular impingement $\cdot$ Hip · Pain management $\cdot$ Sports medicine

\begin{abstract}
A 17-year-old Caucasian male high school baseball catcher presented with bilateral hip and groin pain of insidious onset over a 4-year period. Following appropriate physical exam and radiographic testing, bilateral labral tears secondary to cam type femoroacetabular impingement was diagnosed. Arthroscopic corrective surgeries were performed 4 months apart by a single specialized sports medicine orthopedic surgeon. One hip arthroscopic procedure utilized a well-padded perineal traction post and was associated with neuropraxic complications during recovery. The second hip arthroscopic procedure was performed with the table in $15^{\circ}$ of Trendelenburg for traction without a perineal traction post and no neuropraxia was noted during recovery. The patient reported that outcomes and postoperative radiographic studies were similar bilaterally; however, perineal nerve neuralgias with the second hip arthroscopy were not reported. While other studies have described the use of post-less traction for mitigation of perineal nerve compression during hip arthroscopic procedures, this crossover observational report is the first to the authors' knowledge demonstrating both neuropraxia and lack of neuropraxia in a single patient undergoing the same procedure bilaterally where the only difference is the usage of perineal traction posts.




\section{Case Reports in Orthopedic Research}

\section{Introduction}

Femoroacetabular impingement (FAI) is an aberrant outgrowth of the femoral neck, the acetabular rim, or both and is becoming a more commonly diagnosed cause of anterior hip and groin pain [1]. Treatment of FAI with hip arthroscopy has been shown to provide superior results when compared to open treatment in regard to pain relief and rates of return to sports [2-4]. Surgical treatment of FAI and associated labral tears with hip arthroscopy requires significant mechanical traction to separate the femoral head from the acetabulum to allow for the passage of the arthroscope and instruments into the joint space. Historically, a well-padded perineal post was utilized for countertraction during hip arthroscopy which was associated with compression type injuries to the surrounding neural structures and other soft tissues, reaching complication rates of $73 \%$ postoperatively [5]. Recently, several studies have described methods to mitigate soft tissue and nerve injuries and traction complications associated with perineal post usage during hip arthroscopy with the use of with friction mats, increase in post size and padding, and securing the patient to the table with straps [6-9]. One of these new post-less methods is the use of Trendelenburg positioning as countertraction.

\section{Case Report}

A 17-year-old Caucasian male athlete presented to the orthopedic clinic with a 4-year history of bilateral hip and groin pain of insidious onset. Following physical exam, bilateral cam type FAI was suspected and confirmed radiographically. Figure 1 is the diagnostic pelvic radiograph.

The patient underwent left hip arthroscopic correction first after a failed trial of physical therapy. Figure $2 \mathrm{a}$ and $\mathrm{b}$ represent positioning with a perineal post. The figure demonstrates a perineal post; however, the post used in the case was well padded. Figure $3 \mathrm{a}$ is the visualized left hip joint during the procedure. A tear of the anterior and superior labrum at the chondral labral junction was debrided with an arthroscopic shaver. No anchors were used to correct the labral tear to avoid a possibly out-of-round labral repair. Total traction time with perineal post was 70, with $10 \mathrm{~min}$ where traction was removed intraoperatively to allow the soft tissues to rest. Following removal of traction, the cam lesion was resected.

Postoperatively, the patient was held in recovery due to numbness, bladder dysfunction, and groin pain. He underwent catheterization and was discharged home on the same day as surgery following resolution of urinary symptoms and pain control. When he returned to the clinic 6 weeks postoperatively, no pain with hip motion was noted; however, numbness and paresthesia throughout the groin region persisted. Three months postoperatively, neuropraxia resolved and the patient returned to baseball with no pain in the left hip.

The patient decided to treat his contralateral FAI and right hip arthroscopic correction was planned. Four months following left hip arthroscopy, right hip arthroscopy was performed. The surgical team positioned the patient on the Hana table with an extra safety strap across his chest, secured the lower extremities in traction boots, and placed the table in $15^{\circ}$ of Trendelenburg for countertraction without a perineal post or traction pad. Figure $2 \mathrm{c}$ and $d$ represent the positioning in Trendelenburg. Figure $3 \mathrm{~b}$ is the visualized right hip joint achieved with Trendelenburg positioning. A large tear of the anterior and superior labrum was visualized and repaired using 2 push lock anchors. An arthroscopic shaver was used to remove any loose debris from the joint. The post-less Trendelenburg traction time was $75 \mathrm{~min}$. Following removal of traction, the cam lesion was resected. The patient had no neurologic or urinary 
complications postoperatively. Six weeks postoperatively, the patient reported no hip pain or neuropraxia similar to the first procedure. Three months postoperatively, the patient returned to baseball.

The postoperative pain and physical therapy regimen were identical in both cases. The Harris Hip score was 54 preoperatively and 94 at 3 months postoperatively on the left and 63 preoperatively and 95 at 3 months postoperatively on the right; however, the right hip labral tear was more severe upon direct visualization during arthroscopy.

\section{Discussion}

Rates of hip arthroscopy have increased from 1.2 cases per 10,000 patients in 2004 to 5.58 cases per 10,000 patients in 2009 , with treatment of symptomatic FAI being an increasingly common indication [10]. More recently, studies show that rates have continued to increase $117-150 \%$ from 2007 to 2014 and the percentage of hip arthroscopies for FAI has increased as well $[11,12]$. These trends indicate the need for techniques to minimize patientreported complications such as muscle soreness, bladder dysfunction, scrotal or labial tissue injury, pain, or numbness [5].

Our case reports a 17-year-old Caucasian male high school baseball catcher who underwent both left and right hip arthroscopic labral repair and osteochondralplasty for cam type FAI. The left hip arthroscopy was performed with a well-padded perineal traction post and resulted in subjective neurologic symptoms and urinary dysfunction in the postoperative period that remained for at least 6 weeks consisting of numbness and pain in the perineal region. The surgically more severe right hip arthroscopy performed approximately 4 months later with the table in a $15^{\circ}$ Trendelenburg position was associated with no neurologic or urinary symptoms. By securing the patient in traction boots and placing the surgical table in Trendelenburg without a perineal post, the patient's body weight provides countertraction for appropriate hip distraction. The patient reported in this case report had similar Harris Hip scores and radiographic findings bilaterally. More importantly, however, the patient was subjectively more satisfied with the recovery following the post-less right hip arthroscopy. Several other studies have discussed post-less traction and complications using a Trendelenburg position [6-9]. Complications include iatrogenic injury due to learning a new approach and failure to perform capsulotomy [6-9]. Other post-less traction techniques such as a supine patient with flexion of the lumbar spine and securing the patient to the table with arms crossed with a strap across the chest have been successful for hip arthroscopy procedures as well [6-9]. To the authors' knowledge, however, this is the first reported case where a single patient has acted as his own control to compare subjective outcomes of different hip arthroscopy traction techniques.

This case report has several limitations. The case report involves only 1 patient, which makes it unpowered to make any statistical conclusions; however, the similar surgical procedure of both hips in 1 patient by the same surgical team in a short period of time allows for a unique comparison between the two traction techniques. The urinary issues after the first procedure could have been related to anesthesia medications; however, the same anesthesia team was utilized for both cases. Additionally, any interpretation of nerve symptoms after the second case may have been complicated by the patient's familiarity with the recent left hip surgery.

In conclusion, outcomes between both hips were similar in pain relief, Harris Hip score, radiographically, and in the rate of return to sports activities. However, complications 


\section{Case Reports in Orthopedic Research} www.karger.com/cio

Hagerty and Skelley: Hip Arthroscopy in a Baseball Catcher without the Use of a Perineal Post: A Crossover Case Report

associated with pudendal nerve compression injury were more significant following the procedure in which a perineal traction post was utilized. Additionally, subjective satisfaction of the right-sided post-less arthroscopic procedure was greater than the left-sided procedure. This crossover case report supports the recent literature demonstrating the successful use of post-less traction for hip arthroscopic visualization of the joint space and supports improvement of subjective satisfaction with post-less hip arthroscopic procedures.

\section{Acknowledgements}

We thank the patient, his family, and supporting staff of the University for their help in the preparation of this report.

\section{Statements of Ethics}

The material in this study was reviewed by our Institutional Review Board and they waived processing as no identifying patient information was used. The patient's legal guardian provided written informed consent to publish the case and publish images of the case. The photographed individuals, although completely deidentified, provided consent for inclusion in the technique report.

\section{Conflict of Interest Statement}

The authors have no conflict of interest to disclose.

\section{Funding Sources}

There were no funding sources for this report.

\section{Author Contributions}

Michael P. Hagerty: primary author; Dr. Nathan W. Skelley, MD: supervision of report writing, operating surgeon.

\section{References}

1 Mallets E, Turner A, Durbin J, Bader A, Murray L. Short-term outcomes of conservative treatment for femoroacetabular impingement: a systematic review and meta-analysis. Int J Sports Phys Ther. 2019 Jul;14(4):514-24.

2 Lovett-Carter D, Jawanda A, Hannigan A. Meta-analysis of the surgical and rehabilitative outcomes of hip arthroscopy in athletes with femoroacetabular impingement. Clin J Sport Med. 2020 Jul;30(4):404-11.

3 Nasser R, Domb B. Hip arthroscopy for femoroacetabular impingement. EFORT Open Rev. 2018 Apr;3(4):121-9.

4 Nwachukwu BU, Rebolledo BJ, McCormick F, Rosas S, Harris JD, Kelly BT. Arthroscopic versus open treatment of femoroacetabular impingement: a systematic review of medium- to long-term outcomes. Am J Sports Med. 2016 Apr;44(4):1062-8. 
5 Griffin DR, Dickenson EJ, Wall PD, Achana F, Donovan JL, Griffin J, et al.; FASHIoN Study Group. Hip arthroscopy versus best conservative care for the treatment of femoroacetabular impingement syndrome (UK FASHIoN): a multicentre randomised controlled trial. Lancet. 2018 Jun;391(10136):2225-35.

6 Domb BG, Gupta A, Stake CE, Hammarstedt JE, Redmond JM. Arthroscopic labral reconstruction of the hip using local capsular autograft. Arthrosc Tech. 2014 May;3(3):e355-9.

7 Harris JD. Strategies in managing the labrum. Ann Joint. 2018;3(6):57.

8 Mei-Dan O, McConkey MO, Young DA. Hip arthroscopy distraction without the use of a perineal post: prospective study. Orthopedics. 2013 Jan;36(1):e1-5.

9 Salas AP, Mazek J, Araujo-Reyes D, Gonzalez-Campos M, Castillo-Trevizo A, Garcia JM. The Tutankhamun technique in hip arthroscopy. Arthrosc Tech. 2018 Oct;7(11):e1167-71.

10 Montgomery SR, Ngo SS, Hobson T, Nguyen S, Alluri R, Wang JC, et al. Trends and demographics in hip arthroscopy in the United States. Arthroscopy. 2013 Apr;29(4):661-5.

11 Sing DC, Feeley BT, Tay B, Vail TP, Zhang AL. Age-related trends in hip arthroscopy: a large cross-sectional analysis. Arthroscopy. 2015 Dec;31(12):2307-13.e2.

12 Truntzer JN, Shapiro LM, Hoppe DJ, Abrams GD, Safran MR. Hip arthroscopy in the United States: an update following coding changes in 2011. J Hip Preserv Surg. 2017 Mar;4(3):250-7.
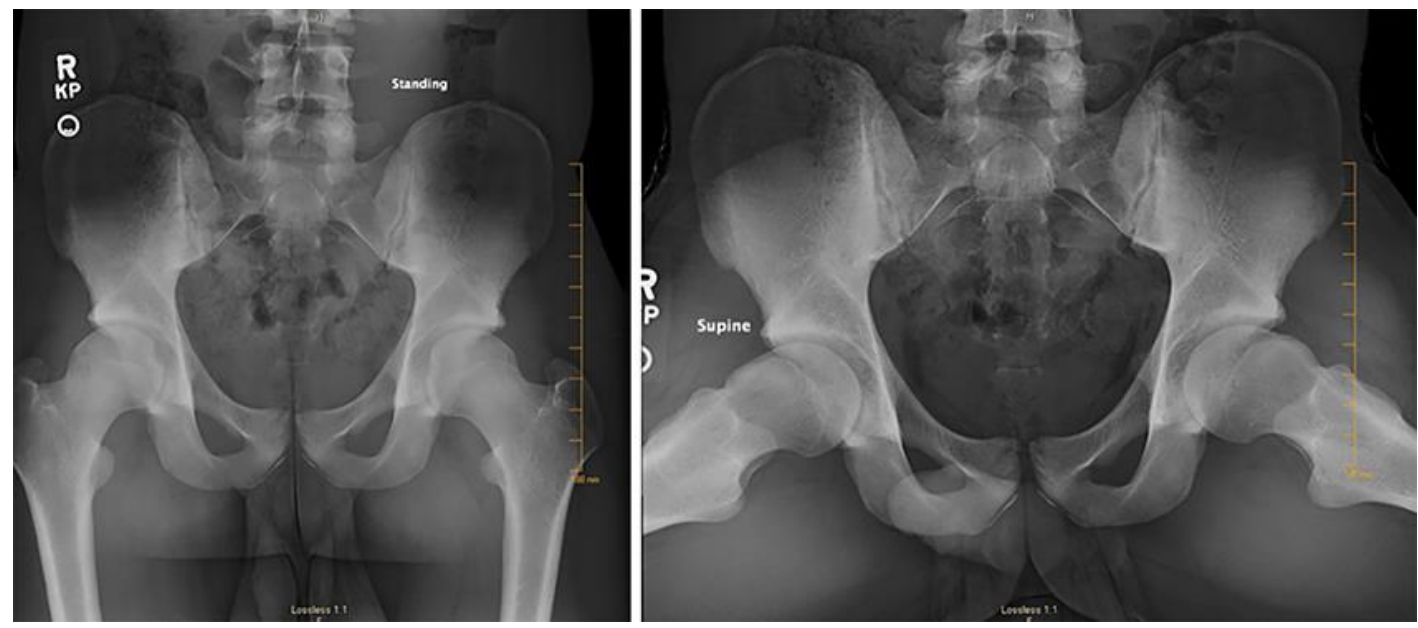

Fig. 1. Diagnostic standing AP and frog-leg pelvic X-rays demonstrating bilateral cam type FAI with otherwise unremarkable radiographic features of a pelvis. 
Case Reports in Orthopedic Research

\begin{tabular}{l|l}
\hline Case Rep Orthop Res 2020;3:94-99 \\
\hline DOI: 10.1159/000509678 & $\begin{array}{l}\text { @ 2020 The Author(s). Published by S. Karger AG, Basel } \\
\text { www.karger.com/cio }\end{array}$ \\
\hline
\end{tabular}

Hagerty and Skelley: Hip Arthroscopy in a Baseball Catcher without the Use of a Perineal Post: A Crossover Case Report
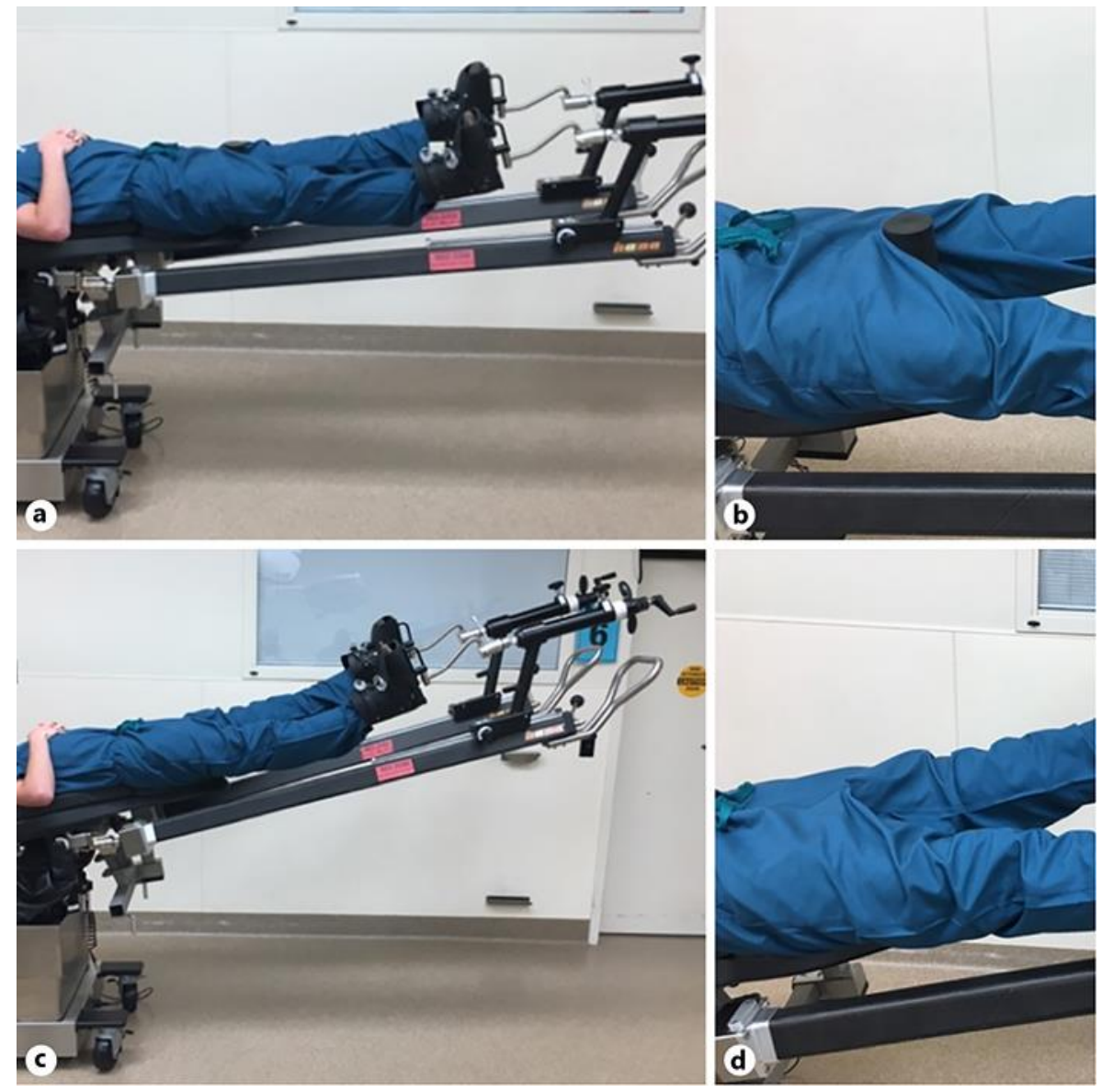

Fig. 2. a-d The positioning of the patient during the hip arthroscopic procedures. $\mathbf{a}, \mathbf{b}$ The traction applied using a perineal post. $\mathbf{c}, \mathbf{d}$ The traction applied as the patient was in Trendelenburg.
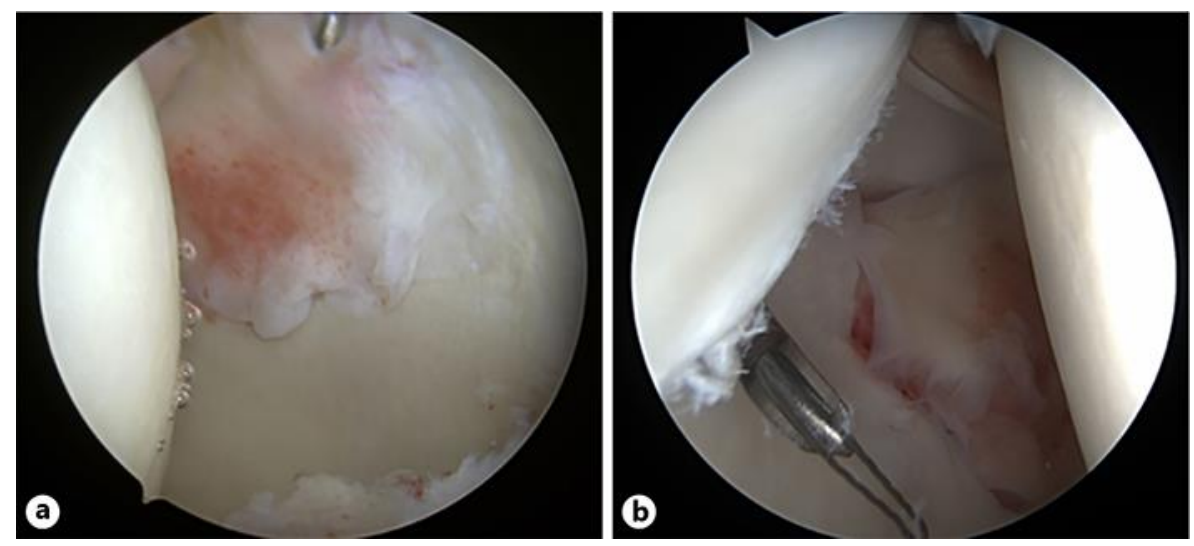

Fig. 3. a, b The appropriate distraction and visualization for labral repair. a The left hip arthroscopy with perineal traction post demonstrating distraction of the hip joint. $\mathbf{b}$ The right hip arthroscopy image without perineal traction post and in $15^{\circ}$ of Trendelenburg position demonstrating appropriate distraction of the hip joint. 